\title{
Turbulent structure and scaling of the inertial subrange in a stratocumulus-topped boundary layer observed by a Doppler lidar
}

\author{
J. Tonttila ${ }^{1,3}$, E. J. O'Connor ${ }^{2,4}$, A. Hellsten ${ }^{2}$, A. Hirsikko ${ }^{2,5}$, C. O'Dowd ${ }^{6}$, H. Järvinen ${ }^{3}$, and P. Räisänen ${ }^{2}$ \\ ${ }^{1}$ Finnish Meteorological Institute, Atmospheric Research Centre of Eastern Finland, P.O. Box 1627, 70211 Kuopio, Finland \\ ${ }^{2}$ Finnish Meteorological Institute, P.O. Box 503, 00101, Helsinki, Finland \\ ${ }^{3}$ University of Helsinki, Department of Physics, P.O. Box 48, 00014, Helsinki, Finland \\ ${ }^{4}$ University of Reading, Reading, UK \\ ${ }^{5}$ Forschungszentrum Jülich GmbH, Institut für Energie-und Klimaforschung: Troposphäre (IEK-8), Jülich, Germany \\ ${ }^{6}$ School of Physics and Centre for Climate \& Air Pollution Studies, Ryan Institute, National University of Ireland Galway, \\ University Road, Galway, Ireland
}

Correspondence to: J. Tonttila (juha.tonttila@fmi.fi)

Received: 29 August 2014 - Published in Atmos. Chem. Phys. Discuss.: 18 September 2014

Revised: 24 April 2015 - Accepted: 9 May 2015 - Published: 27 May 2015

\begin{abstract}
The turbulent structure of a stratocumulus-topped marine boundary layer over a 2-day period is observed with a Doppler lidar at Mace Head in Ireland. Using profiles of vertical velocity statistics, the bulk of the mixing is identified as cloud driven. This is supported by the pertinent feature of negative vertical velocity skewness in the sub-cloud layer which extends, on occasion, almost to the surface. Both coupled and decoupled turbulence characteristics are observed. The length and timescales related to the cloud-driven mixing are investigated and shown to provide additional information about the structure and the source of the mixing inside the boundary layer. They are also shown to place constraints on the length of the sampling periods used to derive products, such as the turbulent dissipation rate, from lidar measurements. For this, the maximum wavelengths that belong to the inertial subrange are studied through spectral analysis of the vertical velocity. The maximum wavelength of the inertial subrange in the cloud-driven layer scales relatively well with the corresponding layer depth during pronounced decoupled structure identified from the vertical velocity skewness. However, on many occasions, combining the analysis of the inertial subrange and vertical velocity statistics suggests higher decoupling height than expected from the skewness profiles. Our results show that investigation of the length scales related to the inertial subrange significantly complements the analysis of the vertical velocity statistics and enables a more confident interpretation of complex boundary layer structures using measurements from a Doppler lidar.
\end{abstract}

\section{Introduction}

Properties of the turbulent variations in vertical velocity, as well as the scaling related to that variability, are important aspects for understanding boundary layer evolution, transport of momentum and thermodynamical properties. These aspects are tightly coupled to the formation and evolution of boundary layer clouds, which in turn strongly affect the radiation budget of the Earth's surface and thus the climate.

Measurements of the turbulent fluctuations of vertical wind in cloud-topped and clear-sky boundary layers as well as inside boundary layer clouds have been performed for decades, typically making use of in situ measurement devices mounted on research aircraft (e.g. Duynkerke et al., 1995; Nicholls, 1984, 1989). Unlike in situ sensors, active remote-sensing instrumentation based at the surface has the significant advantage of being able to routinely measure the velocity profile simultaneously at many levels. A variety of instruments have been employed for this task, such as UHF wind profilers (e.g. Gossard et al., 1998; Jacoby-Koaly et al., 2002), SODARs (e.g. Kouznetsov et al., 2007), Doppler cloud radars (e.g. Shupe et al., 2012) and combinations of these (e.g. Norton, 2006).

Doppler lidars have the necessary high spatial and temporal resolution to derive turbulent properties (Gal-chen et al., 1992; Banakh et al., 1999); recent developments in this field have resulted in robust low-powered instruments designed to operate continuously and autonomously. They are ideal for boundary layer applications, for which they have sufficient 
sensitivity. Since stratocumulus (Sc)-topped boundary layers cover a significant portion of the globe, there have been numerous remote-sensing investigations of them in both marine and continental environments (e.g. Babb and Verlinde, 1999; Duynkerke et al., 1995; Frisch et al., 1995; Hogan et al., 2009; Kollias and Albrecht, 2000; Lothon et al., 2006; Moyer and Young, 1991).

In this article we investigate the scaling of turbulent eddies in a stratocumulus-topped boundary layer and its transition between different mixed-layer structures. The analysed observations cover a boundary layer exhibiting marine characteristics, with both solid and broken cloud structure in the overlying stratocumulus deck. Doppler lidar measurements are used to analyse the vertical velocity field in the boundary layer below the cloud. The aerosol particles in the marine environment provide an ideal tracer for the Doppler lidar and are present in sufficient quantities to provide measurements at high spatial and temporal resolution with good sensitivity throughout the entire vertical extent from near the surface up to cloud base. Robust signal and high resolution allow for a Fourier analysis of vertical velocity as a function of height. The resulting power spectra are used to investigate the relative scaling of the turbulent eddies and contrast them with the bulk statistical properties of the vertical velocity distribution. The high-resolution, vertically resolved spectral analysis along with complementing profile data of the turbulence statistics is only possible using vertically pointed Doppler lidars. This provides a unique and self-consistent data set to study the relationships between vertical velocity statistics, the scaling of the turbulent eddies, the source of kinetic energy in the mixed layer and the general vertical structure of the boundary layer. Unless otherwise mentioned, our analysis focuses on the properties of the below-cloud portion of the boundary layer only, in contrast to e.g. Ghate et al. (2014), who employed a combination of data from a Doppler lidar and a cloud radar.

Inspired through recent studies by Hogan et al. (2009) and Harvey et al. (2013), who provided observational evidence of how to identify and isolate the cloud-driven mixing from surface-based mixing in a stratocumulus-topped boundary layer based on the sign of the skewness of vertical velocity, we hypothesise that the scaling of the inertial subrange determined from the spectral analysis can be used as an additional diagnostic to identify the sources of turbulent mixing. As the sign of the skewness indicates the direction of the flux of turbulent kinetic energy (TKE), the cloud-driven and surface-based layers can be seen to exhibit opposite and thus competing effects, depending on the relative strength of the sources of TKE and the stability of the mixed layer. It is then also expected that the scaling of the inertial subrange changes according to the mixed-layer structure. This forms the basis for comparing the kinetic statistics with the scaling of the turbulent eddies, which we attempt to use in synergy to complement the individual diagnostics and thus to provide confirmation for the conclusions drawn about the boundary
Table 1. Doppler lidar operating parameters for the vertical stare mode during the deployment at Mace Head. System parameters for an individual radial measurement in the DBS mode, where different, are given in parentheses.

\begin{tabular}{ll}
\hline Wavelength & $1.5 \mu \mathrm{m}$ \\
Pulse repetition rate & $15 \mathrm{kHz}$ \\
Nyquist velocity & $19.6 \mathrm{~m} \mathrm{~s}^{-1}$ \\
Sampling frequency & $50 \mathrm{MHz}$ \\
Points per range gate & 10 \\
Pulses averaged & $150000(300000)$ \\
Range resolution & $30 \mathrm{~m}$ \\
Integration time & $10 \mathrm{~s}(20 \mathrm{~s})$ \\
Pulse duration & $0.2 \mu \mathrm{s}$ \\
Lens diameter & $6 \mathrm{~cm}$ \\
Divergence & $33 \mu \mathrm{rad}$ \\
Focus & $1 \mathrm{~km}$ \\
Antenna & monostatic optic-fibre \\
& coupled \\
\hline
\end{tabular}

layer structure, solely based on retrievals from the Doppler lidar instrument. We will also briefly discuss how the scaling of the inertial subrange and its evolution with changing boundary layer structure may affect scale-dependent lidarbased retrievals.

The layout of this paper is as follows: Sect. 2 describes the instrumentation and the main analysis methods. A description of the synoptic situation and key features during the analysed period is given in Sect. 3. The results obtained for turbulence statistics and the scaling of the inertial subrange are given in Sect. 4, followed by concluding remarks.

\section{Methodology}

\subsection{Instrument}

The data for this study were obtained from a coherent heterodyne pulsed Doppler lidar (production no. 34), owned by the Finnish Meteorological Institute and deployed at Mace Head, on the west coast of Ireland $\left(53^{\circ} 19^{\prime} \mathrm{N}, 9^{\circ} 53^{\prime} \mathrm{W}\right)$, from 16 February to 27 March 2012 (Hirsikko et al., 2014). Operating specifications for the Doppler lidar are given in Table 1. Initial data points are oversampled at $3 \mathrm{~m}$ resolution, and 10 points are then combined to give a final spatial resolution of $30 \mathrm{~m}$. A total of 320 gates gives a maximum range of $9.6 \mathrm{~km}$. The temporal resolution can be as high as $1 \mathrm{~s}$. However, to obtain good sensitivity, it is usually necessary to integrate further, since useful signals are only obtained in the presence of a reasonable aerosol load or when clouds are present.

The instrument was operated predominantly in the zenithpointing stare mode, interspersed with a wind scan sequence every $10 \mathrm{~min}$ (giving six wind profiles per hour). For this campaign, an integration time of $10 \mathrm{~s}$ was selected for the vertical stare mode, sufficiently long for acquiring profiles 
with reasonably small uncertainties while short enough for deriving turbulent properties.

As standard, the Doppler lidar provides profiles of signalto-noise ratio (SNR), uncalibrated attenuated backscatter coefficient and radial Doppler velocity. Post-processing (Hirsikko et al., 2014) then applies background and focus corrections to the signal and provides calibrated attenuated backscatter coefficient profiles, together with uncertainties in the signal, attenuated backscatter and Doppler velocity derived using an approximation to the Cramér-Rao lowerbound method (Rye and Hardesty, 1993) given in O'Connor et al. (2010).

The horizontal wind profiles were obtained using a threebeam Doppler beam swinging (DBS) technique. The wind scan sequence consisted of three consecutive rays, one pointing to the zenith, and two orthogonal rays at $20^{\circ}$ from the zenith (one pointing north, one pointing east). Vertical profiles of horizontal winds can then be obtained through trigonometry from radial velocities under appropriate conditions (e.g. Koscielny et al., 1984). As noted in Table 1, to reduce uncertainties in the retrieved horizontal winds, the integration time for each ray in the wind scan sequence was twice the integration time for an individual ray in the zenithpointing mode. A single vertical profile of horizontal winds therefore took about $60 \mathrm{~s}$ to obtain.

Data quality is provided directly by examining SNR (after applying any background correction). The threshold is determined based on the acceptable uncertainty for a given application. For vertically pointing data, the selected threshold of $-21 \mathrm{~dB}$ for SNR is equivalent to an uncertainty of about $0.05 \mathrm{~m} \mathrm{~s}^{-1}$ for this particular Doppler lidar instrument in this configuration. The Doppler lidar attenuated backscatter coefficient can additionally be calibrated according to a procedure introduced by O'Connor et al. (2004). In this method, the integral of attenuated backscatter from a nearly non-drizzling cloud base through to infinity is set equal to $1 /(2 \eta S)$, where $\eta$ is the multiple scattering factor and $S$ is the lidar ratio. Both $\eta$ (close to 1$)$ and $S(20 \mathrm{sr}$ ) are assumed constant and known for this instrument and lidar wavelength in stratocumulus clouds (Westbrook et al., 2010). Drizzling clouds are screened from the calibration procedure by a non-drizzling condition. There it is required that attenuated backscatter coefficient values at $250 \mathrm{~m}$ below the cloud base are 10 times smaller than the attenuated backscatter coefficient inside the liquid cloud (O'Connor et al., 2004). The uncertainty in the calibration method is $20 \%$.

\subsection{Vertical velocity analysis}

The Doppler lidar produces vertical velocity profiles at $10 \mathrm{~s}$ resolution. Turbulent properties were derived from statistical properties of the vertical velocity distribution over longer intervals. The properties are computed at every range gate of the lidar, giving a high-resolution vertical profile of each turbulent property.

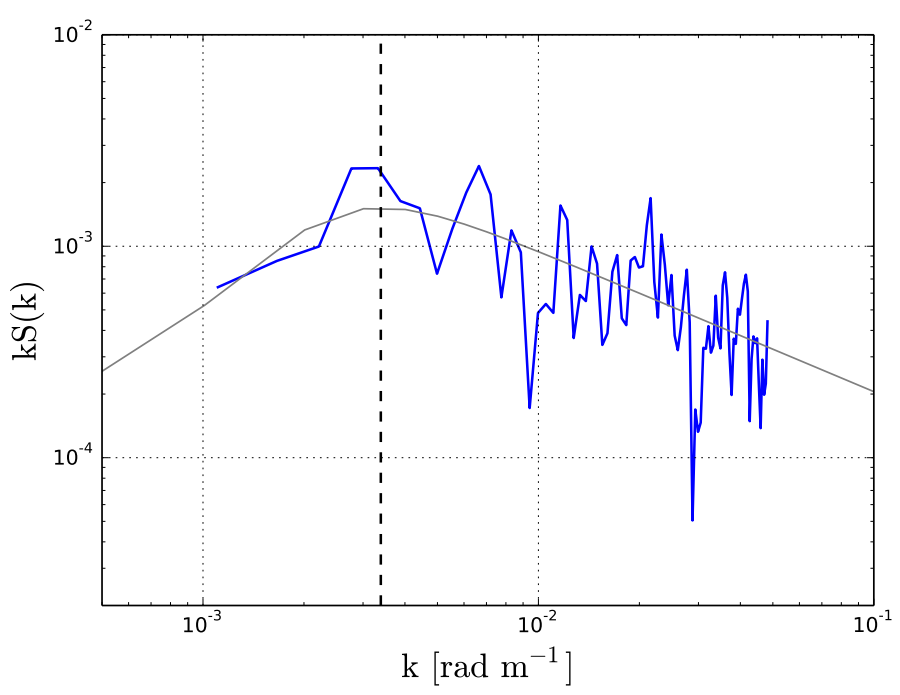

Figure 1. An example of a vertical velocity power spectral density $(S)$ as a function of the wave number $k$ (blue line) taken from roughly the middle of the well-mixed layer on 25 February at 04:00 UTC. The thin grey line represents the fit from Eq. (3). The black dashed line shows the wave number corresponding to $\lambda_{0}$ in this particular example. The inertial subrange is found for wave numbers above this point.

The second and third moments of the velocity distribution, standard deviation $\sigma_{w}$ and skewness $\gamma_{w}$, are calculated from sequential vertical velocity samples over a $60 \mathrm{~min}$ interval:

$$
\begin{aligned}
\sigma_{w} & =\sqrt{\frac{1}{n} \sum_{i=1}^{N}\left(w_{i}-\bar{w}\right)^{2}} \text { and } \\
\gamma_{w} & =\frac{\frac{1}{n} \sum_{i=1}^{N}\left(w_{i}-\bar{w}\right)^{3}}{\sigma_{w}^{3}}
\end{aligned}
$$

respectively, where $\bar{w}$ is the sample mean vertical velocity and $w_{i}$ is the $i$ th vertical velocity sample. Due to the interspersed sampling of the horizontal wind every $10 \mathrm{~min}, n$ for the $60 \mathrm{~min}$ period is in practice about 320 .

The vertical velocity power spectrum is used to identify the range of scales over which turbulent mixing predominates, commonly known as the inertial subrange. This is accomplished by finding the transition wavelength $\lambda_{0}$ at which the spectral density peaks and the slope deviates from the expected $-5 / 3$ power law, as shown by an example from the analysed data in Fig. 1. The spectral model by Kristensen et al. (1989), also applied by Lothon et al. (2009), is used to identify the transition wavelength, an example of which is also shown in Fig. 1. The model-based spectral intensity as a function of the wave number $k$ is given by

$$
S(k)=\frac{\sigma_{w}^{2} l}{2 \pi} \frac{\left(3+8\left(\frac{l k}{a}\right)^{2 \mu}\right)}{3\left(1+\left(\frac{l k}{a}\right)^{2 \mu}\right)^{\frac{5}{6 \mu}+1},}
$$




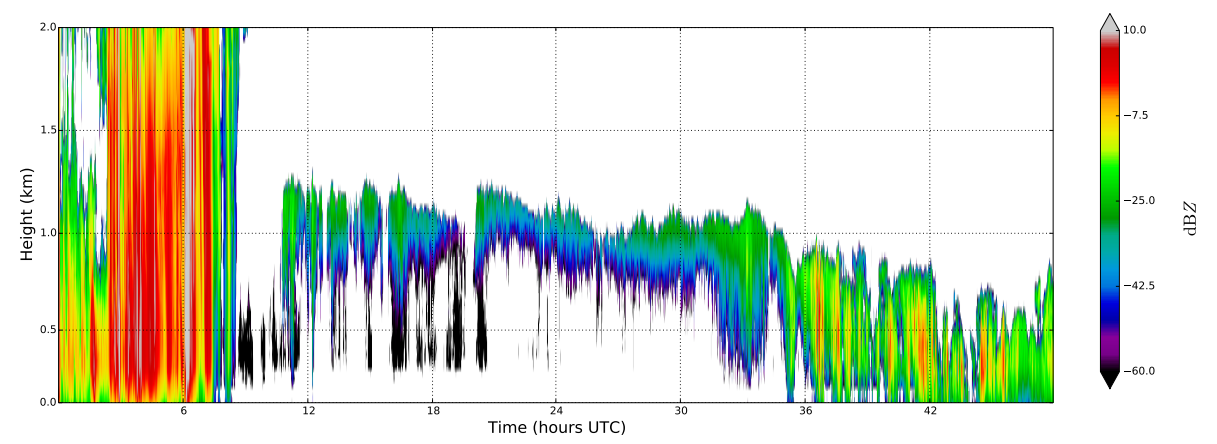

Figure 2. Radar backscatter cross section over the 2-day period used in the analysis.

where $\mu$ controls the curvature of the spectrum and the parameter $l$ is the integral scale of vertical velocity along the horizontal flow trajectory. In this model, $l$ can be expressed as a function of $\lambda_{0}$ and $\mu$ (i.e. inverse solution of Eq. (3) in Lothon et al., 2009). Furthermore, $a$ is given as a function of $\mu$ :

$a(\mu)=\frac{\pi \mu \Gamma\left(\frac{5}{6 \mu}\right)}{\Gamma\left(\frac{1}{2 \mu}\right) \Gamma\left(\frac{1}{3 \mu}\right)}$,

where $\Gamma$ is the gamma function. In our analysis we set $\mu=$ 1.5 , as it provided a good match with most of the observed spectra. This yields $a \approx 0.69$.

The transition wavelength can be normalised by the depth of the mixed layer $z_{i}$ to give

$L_{0}=\frac{\lambda_{0}}{z_{i}}$.

We create the power spectrum from consecutive velocity samples over a $30 \mathrm{~min}$ interval. The parameter $z_{i}$ is estimated for each derived spectrum as the local mixed-layer depth. For decoupled mixed layers, this is estimated as the difference between the boundary layer top and the estimated decoupling height taken as the level where vertical velocity skewness changes sign, typically from positive in the lower layers to negative towards the cloud (e.g. Hogan et al., 2009; Ghate et al., 2014). Here, the boundary layer top is taken as the cloud-top height derived from coincident Doppler cloud radar measurements (the $35.5 \mathrm{GHz}$ MIRA).

In this paper, we also utilise the turbulent dissipation rate, which is derived from the high temporal resolution vertical velocities (O'Connor et al., 2010):

$\epsilon=2 \pi\left(\frac{2}{3 a_{k}}\right)^{\frac{3}{2}} \sigma_{\overline{\mathrm{v}}}^{3}\left(L^{2 / 3}-L_{1}^{2 / 3}\right)^{-3 / 2}$,

where $a_{k}=0.55$ is the Kolmogorov constant for onedimensional wind spectra, $\sigma_{\overline{\mathrm{v}}}$ is the standard deviation of the mean velocity over $N$ sequential velocity samples, $L$ is the spatial length scale corresponding to the number of samples used for calculating $\sigma_{\overline{\mathrm{v}}}$ and $L_{1}$ is the length scale appropriate for an individual sample. In this study we use $N=12$ samples, which corresponds to an averaging interval of $2 \mathrm{~min}$. The length scales are then computed as $L=N U t$, where $U$ is the horizontal wind speed as measured by the Doppler lidar DBS scan sequence, and $t$ is the integration time for one ray. Note that $\sigma_{\overline{\mathrm{v}}}$ is calculated over a much shorter time interval than $\sigma_{w}$ (2 min vs. $\left.60 \mathrm{~min}\right)$.

\section{Meteorological conditions and general features}

The stratocumulus-topped marine boundary layer studied here was observed over Mace Head, Ireland, during 2425 February 2012. There was a large area of high pressure to the south of Ireland, extending west from France out into the Atlantic. To the north were adjacent centres of low pressure west of Iceland and over Scandinavia. The predominant flow over Ireland was from a westerly direction. Our analysis concentrates on the stratocumulus clouds emerging after the overpass of a weak remnant of the tail end of a precipitating cold front, extending from an occlusion associated with the low-pressure centre that had moved from the North Atlantic to Eastern Europe by 18:00 UTC on 24 February. The passage of the front over Mace Head occurred during the early hours of 24 February, and, by 08:00 UTC, the rain at the surface associated with the front died out. The remaining midand high-level clouds associated with the frontal area diminished by around 11:00 UTC. Figure 2 shows the time-height cross section of the analysed period from a Doppler cloud radar with the stratocumulus clouds emerging after the front has passed. The passage of the front is also evident in the horizontal wind field observed by the Doppler lidar (Fig. 3) as wind speeds decrease from 15 to $8 \mathrm{~m} \mathrm{~s}^{-1}$. This period between approximately 08:00 to 11:00 UTC appears virtually non-turbulent in the observations of the lower atmosphere; the surface front has already passed, but the boundary layer is still influenced by the presence of the frontal zone at upper levels. The clouds associated with the frontal zone at upper levels are still present. Due to a moderate horizontal flow from the north-west (approximately $8 \mathrm{~m} \mathrm{~s}^{-1}$; Fig. 3), 

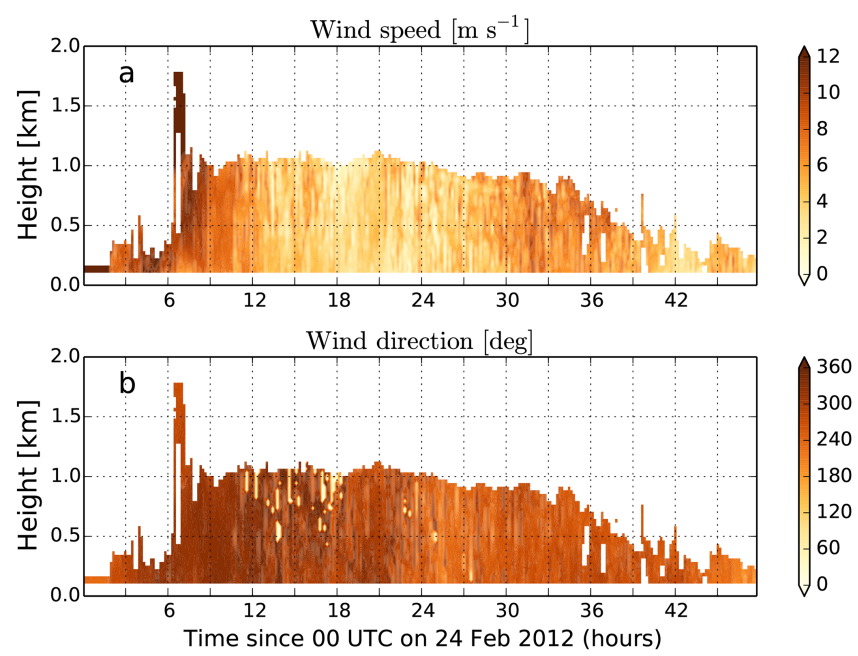

Figure 3. Profiles of (a) the horizontal wind speed and (b) direction over the analysed 2-day period.
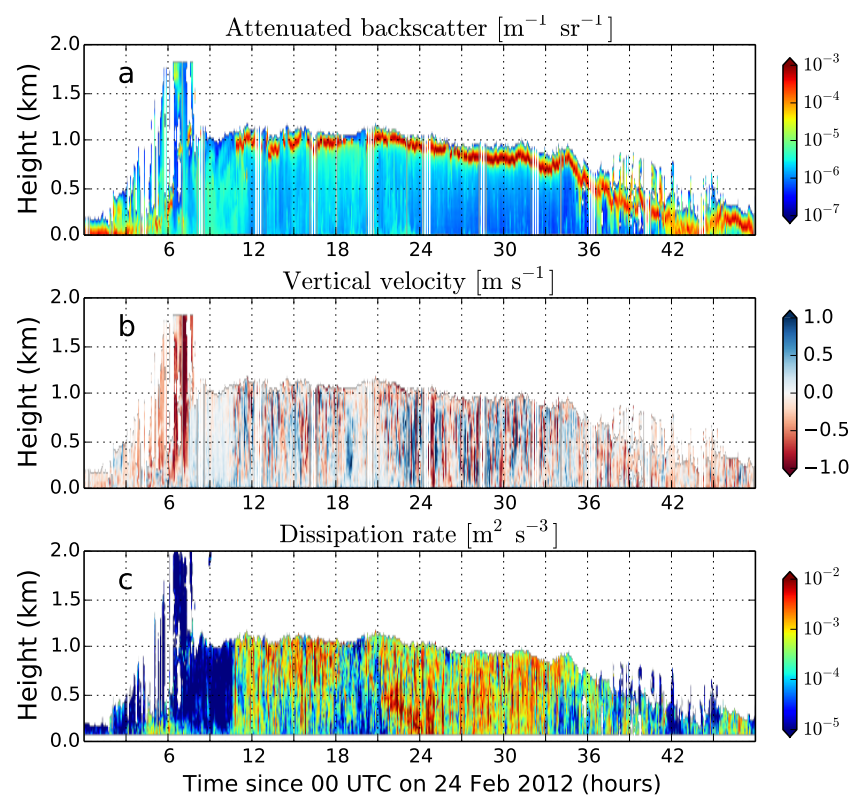

Figure 4. (a) Lidar backscatter, (b) Doppler velocity and (c) turbulence dissipation rate.

a rather shallow surface-based mixed layer with marine characteristics is expected over the observation site. The turbulence characteristics observed with the lidar are shown in Figs. 4 and 5, in which the profiles of $\sigma_{w}$ and $\epsilon$ indeed indicate the existence of a very shallow mixed layer close to the surface. Above $150 \mathrm{~m}$, the turbulence is very weak with $\sigma_{w} \leq 0.1 \mathrm{~m} \mathrm{~s}^{-1}$ and very low $\epsilon$. At these heights, the properties of the flow are more reminiscent of the free-tropospheric conditions rather than the boundary layer, although the layer still contains enough particles for a relatively strong lidar signal up to about $1000 \mathrm{~m}$ height.
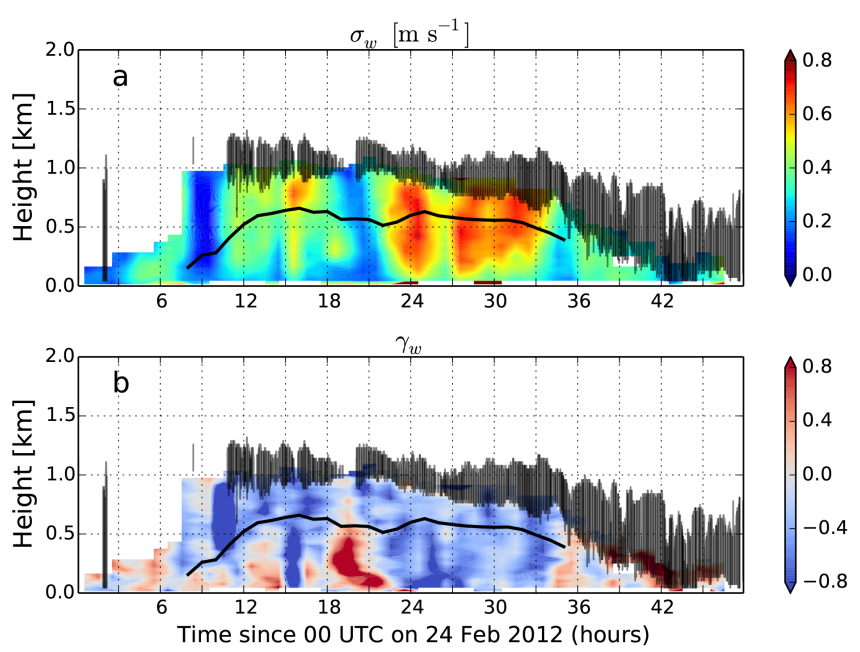

Figure 5. (a) Standard deviation and (b) skewness of vertical velocity. The black shading shows the location of the cloud layer, where cloud base is diagnosed from the lidar measurements and radar measurements are used to infer cloud-top height. The black line gives the lifting condensation level estimated from surface temperature and humidity measurements.

Later in the afternoon of 24 February, the north-westerly horizontal flow weakens gradually to about $4 \mathrm{~m} \mathrm{~s}^{-1}$ and remains low until 27:00 UTC (counting from 00:00 UTC on 24 February), when the wind speed starts to increase. The base of the Sc layer is at approximately the height of $1000 \mathrm{~m}$ on 24 February, as observed by both the lidar and radar (Figs. 2 and 4). During the 22:00-32:00 UTC period, the cloud base height decreases gradually from $1000 \mathrm{~m}$ to about $800 \mathrm{~m}$. The 27:00-32:00 UTC period, however, features a rather uniform cloud structure with almost constant cloud base height. As shown later, this provides an interesting counterpart for the broken cloud structure seen in the afternoon of 24 February.

Cloud-topped boundary layers can exhibit many different structural types (Lock et al., 2000; Harvey et al., 2013). The boundary layer mixing is defined as coupled when the cloud layer is directly associated with the turbulent mixing originating from the surface due to buoyant or mechanical turbulence generation, or when turbulent mixing driven by cloudtop radiative cooling extends to the below-cloud mixed layer and even all the way down to the surface (Garrat, 1992). The mixing in the boundary layer is defined as decoupled when the cloud-driven mixing is not associated with the surface or surface processes. Typically, mixing in and below Sc layers is driven by the long-wave radiative cooling of the Sc deck itself and is important in maintaining the Sc cloud layer through the vertical transport of moisture, especially when there is no substantially strong turbulent vertical transport driven by surface processes.

A broken cloud deck is evident during the afternoon of 24 February, caused by breaks between the cellular struc- 
ture in the stratocumulus advected over the site. The cloud base height shows some variation over time, although mainly less than $150 \mathrm{~m}$, with less variation in cloud-top height. We will show later in Sect. 4 that the daytime broken clouds on 24 February are associated with decoupled mixing, while the cloud deck in the morning of 25 February can be regarded as coupled yet still cloud driven.

A longer break in the low-level clouds occurs from 18:00 to 20:00 UTC, which coincides with cirrus clouds emerging over the site. The stratocumulus deck re-emerges when the upper-level cirrus begins to diminish. Unlike the broken field in the cloud deck earlier in the afternoon, which is probably due to internal Sc dynamics, this longer gap appears to be the result of the radiative impact of the cirrus layer above. Christensen et al. (2013) showed that during night-time, on short timescales on the order of a few hours, an upper-level cloud significantly decreases the cloud-top radiative cooling and the liquid water path of the stratocumulus layer. In essence, part of the up-welling long-wave radiation is absorbed and re-emitted downwards by the cirrus and reduces the Sc cloudtop radiative cooling. Without vertical transport of moisture through Sc cloud-top radiative cooling, the Sc layer cannot maintain itself and dissipates. This corresponds very well to our observations, because when the cirrus cloud layer emerging over the Sc deck becomes optically thick it eventually causes the transitory dissipation of the low-level stratocumulus. Once the cirrus layer is no longer optically thick enough, it does not take long for the Sc layer to return.

\section{Turbulence structure in coupled and decoupled cloud-driven mixed layers}

\subsection{Vertical velocity statistics}

The time-height cross sections of the lidar attenuated backscatter, Doppler vertical velocity and the turbulence dissipation rate are shown in Fig. 4, while Fig. 5 shows the vertical velocity statistics $\left(\sigma_{w}\right.$ and $\left.\gamma_{w}\right)$. The latter also shows the location of the cloud layer, where the cloud base is determined from the Doppler lidar using a threshold of $10^{-4} \mathrm{~m}^{-1} \mathrm{sr}^{-1}$ for the attenuated backscatter. The location of the cloud base is not particularly sensitive to thresholds close to this value - e.g. $10^{-5} \mathrm{~m}^{-1} \mathrm{sr}^{-1}$ gives a very similar result. The cloud-top height is diagnosed from the cloud radar, with $50 \mathrm{dBZ}$ used as the threshold for radar reflectivity. Judging by $\sigma_{w}$ and the lidar attenuated backscatter profile, the strongest turbulent variability is generally connected with stratocumulus-topped profiles. It is also evident that $\sigma_{w}$ tends to increase towards the cloud deck throughout the observed period. While relatively intense mixing is observed during the 23:00-32:00 UTC period for the whole depth of the boundary layer (with maximum $\sigma_{w}=0.8 \mathrm{~m} \mathrm{~s}^{-1}$ near the cloud layer and $0.5 \mathrm{~m} \mathrm{~s}^{-1}$ also near the surface), the 11:00-18:00 UTC period shows generally weaker mixing and a more pronounced difference between the near-surface and below-cloud layers. The results imply that the mixing is primarily driven by cloud-top radiative cooling (Lock, 1998; Hogan et al., 2009; Harvey et al., 2013), which is commonly observed in midlatitude marine stratocumulus. The profile of the dissipation rate shown in Fig. 4 supports this conclusion. The collocated Doppler cloud radar observations also indicate considerable turbulent activity within the cloud layer. Other processes that have an impact on the cloud-driven mixing include entrainment, although it is often difficult to separate these processes in remote sensing measurements (Kollias and Albrecht, 2000).

Our interpretation of the boundary layer structure is further supported when examining the skewness profiles in Fig. 5; negative skewness of vertical velocity, which has been shown to indicate cloud-driven mixing (Hogan et al., 2009), is a predominant feature of the below-cloud mixed layer (although it may also occur near the tops of clear-sky surface-driven layers). Moreover, Hogan et al. (2009) noted that cloud-driven mixing in many ways resembles "upside-down" convective mixing, which is supported by the profiles of $\epsilon$ and $\sigma_{w}$ in Figs. 4 and 5. Similar behaviour has also been observed for in-cloud statistics (Frisch et al., 1995; Kollias and Albrecht, 2000).

Figure 6 shows $\sigma_{w}, \gamma_{w}$ and $\epsilon$ as line plot profiles for consecutive $1 \mathrm{~h}$ segments on 24 February (from 11:00 to 18:00 UTC), providing more detailed evidence. While $\sigma_{w}$ is, again, seen to peak near or within the cloud layer, the profiles of $\gamma_{w}$ and $\epsilon$ show the separation between the cloud-driven and surface-based layers on many occasions, with higher $\epsilon$ and negative $\gamma_{w}$ being the dominant features in the upper part of the boundary layer. An analysis based on $\gamma_{w}$ suggests that the cloud-driven layer is decoupled and that the height of decoupling is found at around $500-600 \mathrm{~m}$ at maximum. Unfortunately, we lack collocated thermodynamical profile measurements to further characterise the vertical boundary layer structure. However, surface measurements of temperature and humidity are used to estimate the lifting condensation level (LCL) (Lawrence, 2005) and shown as the black line in Fig. 5. Being clearly below the actual cloud base, this also indicates the existence of a decoupled mixed-layer structure. Moreover, it is noted that operational soundings from Valentia, although about $160 \mathrm{~km}$ to the south from Mace Head, share approximately similar meteorological conditions at least during the afternoon of 24 February and suggest a decoupling height similar to that diagnosed here from the lidar observations after 12:00 UTC. In some cases, Fig. 6 suggests that, for the cloud-driven layer, the depth of influence according to the skewness statistics may penetrate deeper than what would be the actual decoupling height. A case in point is e.g. a sharp increase in $\epsilon$ around $500-600 \mathrm{~m}$ for samples after 15:00 UTC, while the region of negative skewness reaches much lower levels starting from the cloud layer. In contrast to the boundary layer structure seen for the afternoon, Fig. 7 shows a similar plot for 25 February (from 00:00 

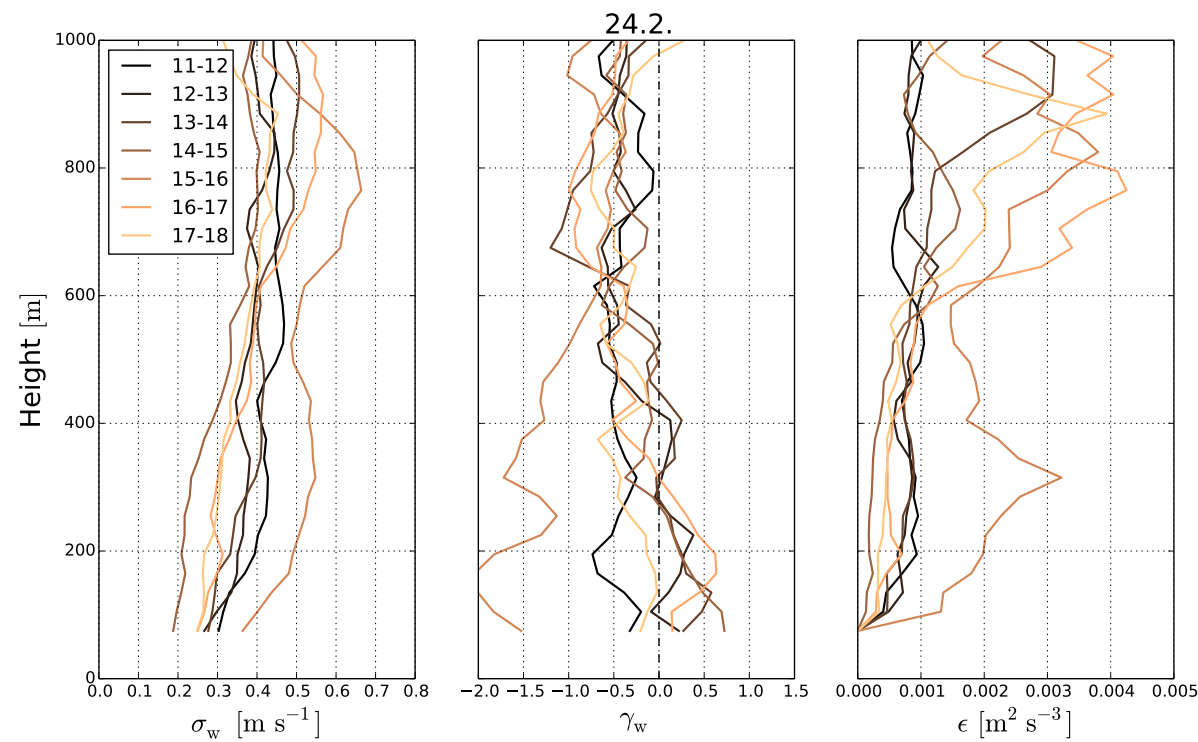

Figure 6. Profiles of the standard deviation $\left(\sigma_{w}\right)$ and skewness $\left(\gamma_{w}\right)$ of vertical velocity and the dissipation rate $(\epsilon)$ for $1 \mathrm{~h}$ segments during the early hours of 24 February.

to 06:00 UTC) for a deep cloud-driven mixed layer: the profiles of $\sigma_{w}$ and $\epsilon$ are characteristic of a well-mixed boundary layer. A similar conclusion can be made for $\gamma_{w}$ as well, noting that its features are quite the opposite (both in sign and the shape of the vertical profile) to what would be expected if the mixing was driven by surface-based buoyancy production. The LCL shown in Fig. 5 (after 24:00 UTC) is still somewhat lower than the actual cloud base, but they appear to merge towards the morning hours, indicating a gradual reduction in the degree of decoupling.

The evolution of the negative $\gamma_{w}$ region suggests a difference in the depth of the cloud-driven layer between the afternoon of 24 February and the night/early morning of 25 February, which is evident when comparing Figs. 6 and 7. A region of weakly positive $\gamma_{w}$ extends upwards from the surface during the afternoon of 24 February, suggesting the growth of a surface-based mixed layer, although $\sigma_{w}$ is rather weak for this region. The growth of the surface-based layer reduces the depth of the cloud-driven portion of the boundary layer with negative $\gamma_{w}$. No such layer of positive skewness is visible on 25 February. Assuming that the vertical mixedlayer interface can be found by examining the sign of the vertical velocity skewness, the maximum decoupling height is found during 12:00-15:00 UTC, while during the night of 25 February the cloud-driven mixing is strong enough to support a coupled layer. The results in Figs. 6 and 7 are very reminiscent of those presented in Ghate et al. (2014) for similar situations. Detecting the decoupling height is discussed further in Sect. 4.2.

What then causes the surface-based layer to expand during the 12:00-18:00 UTC period? Potential factors are identified and explained next. The broken cloud structure seen in the early afternoon could allow increased direct solar surface heating of the coastal observation site, thus promoting growth of the surface mixed layer. However, due to the close proximity to the ocean and the low angle of the wintertime sun, this effect is most likely weak. The broken cloud structure, as well as direct solar radiation modifying the temperature profile, could also decrease the production of the turbulent kinetic energy at the top of the cloud layer. However, $\epsilon$ remains quite high within the upper portion of the clouddriven mixed layer throughout this period. In addition, the surface-based layer starts to extend by noon, before there are any obvious gaps in the cloud layer. The key feature to note is that the horizontal wind speed starts to decrease around noon and continues to do so during the afternoon (Fig. 3). With the onshore wind weakening, there is more competition between the shallow marine surface-driven layer and the deeper surface-driven mixed layer generated inland; the surfacedriven mixed layer above this coastal location is influenced by both weak heat fluxes from the ocean surface and relatively strong heat fluxes from the land, at least during the day. Furthermore, Fig. 3 suggests some height-dependent fluctuation of the horizontal wind during this period. The fluctuations might act to trigger periods where the stability near the surface is reduced, allowing a surface-based TKE production to affect a deeper layer. In particular, we cannot rule out the possibility of advection of cooler air aloft, which could potentially act as a driver for such events. At the same time, this could also act to slightly increase the stability in the upper portion of the boundary layer, further contributing to the relative strength and extent of the cloud-driven and surface-based mixed layers. It should be noted that the surface-based TKE production is primarily quite weak throughout the period, as 

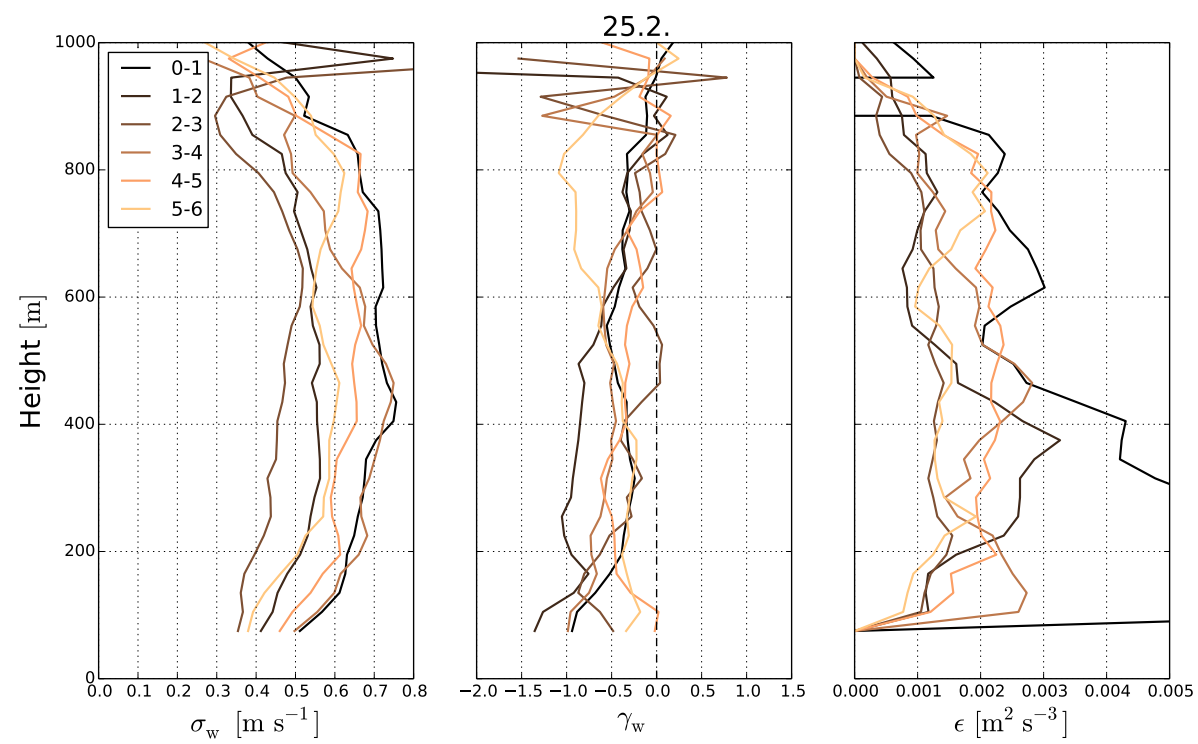

Figure 7. Profiles of the standard deviation $\left(\sigma_{w}\right)$ and skewness $\left(\gamma_{w}\right.$ of vertical velocity and the dissipation rate $(\epsilon)$ for $1 \mathrm{~h}$ segments during the early hours of 25 February.

indicated by the profiles of $\epsilon$ in Fig. 6. Moreover, profiles of $\sigma_{w}$ suggest that the degree of decoupling is likely moderate, as in most cases there are no particularly sharp changes in the vertical. If considerable surface-based turbulence generation along with the cloud-driven mixing were present, one might expect a minimum of TKE and thus the dissipation rate near the decoupling height. However, this is not clearly seen in our measurements during the afternoon of 24 February, which can be attributed to the general weakness of the surface-driven mixing. Nevertheless, the decoupled structure can be identified, as further illustrated by the scaling of the inertial subrange in the next section. This supports the idea that changes in the stability of the layers would be the reason for changes in the relative depths of influence for the surface-based and cloud-driven layers. It is then understood that a coupled well-mixed layer is formed in this case only with sufficiently intense TKE generation by the cloud layer, which is shown by $\sigma_{w}$ in Fig. 7.

After 18:00 UTC, $\epsilon$ is reduced significantly, which appears to be coupled with the dissipation of the cloud-driven turbulence and the cloud layer itself. A factor that likely contributes to this is the reduced radiative cooling of the Sc cloud top due to the presence of an overlying cirrus layer between about 17:00 and 21:00 UTC (not shown). This period is characterized by positive $\gamma_{w}$ near the surface. However, the significance of this signal is questionable because the width of the vertical velocity distribution becomes very narrow due to weak turbulence at this time. There are also slight changes in wind direction and speed which might cause subtle variations in the vertical velocity moments during this time. As the cirrus layer departs after a couple of hours and the Sc layer reappears, the cloud-driven mixed layer is quickly regener- ated and starts to encroach into the practically non-turbulent surface layer.

\subsection{Scaling of the inertial subrange}

We now investigate the scaling of the inertial subrange in the stratocumulus-topped periods and relate those results to the differences in the turbulence statistics described in the previous section. The relative scaling of the inertial subrange $L_{0}$ is analysed according to Eq. (5) at two height levels in the boundary layer: one near the cloud base and one in the surface-based regime (yet no lower than $100 \mathrm{~m}$ to avoid spurious data), based on the estimated decoupling height. We attempt to estimate the decoupling height by finding the level where vertical velocity skewness changes sign, typically from positive to negative when ascending towards the cloud layer, as seen in Fig. 5. The scaling of $L_{0}$ at the two sampling levels is presented in Fig. 8, together with the estimated decoupling height at the interface between the two regimes. In addition, an alternative version of the surface-based $L_{0}$ is provided for comparison ("Surface alt." in Fig. 8), in which the depth of the cloud-driven layer is used instead as the normalising factor $z_{i}$. Figure 8 also shows the below-cloud mean horizontal wind speed, and black shading indicates the presence of cloud (cloud base is retrieved from the lidar, and cloud top is from the cloud radar as in Fig. 5). As the sign of the skewness indicates the direction of the turbulent flux of kinetic energy associated with the mixing, it is expected that the scaling of the inertial subrange would also present differences according to the mixed-layer depth and the source of TKE.

It is not always possible to derive $L_{0}$; the vertical velocity power spectra can be very noisy in regions with low li- 

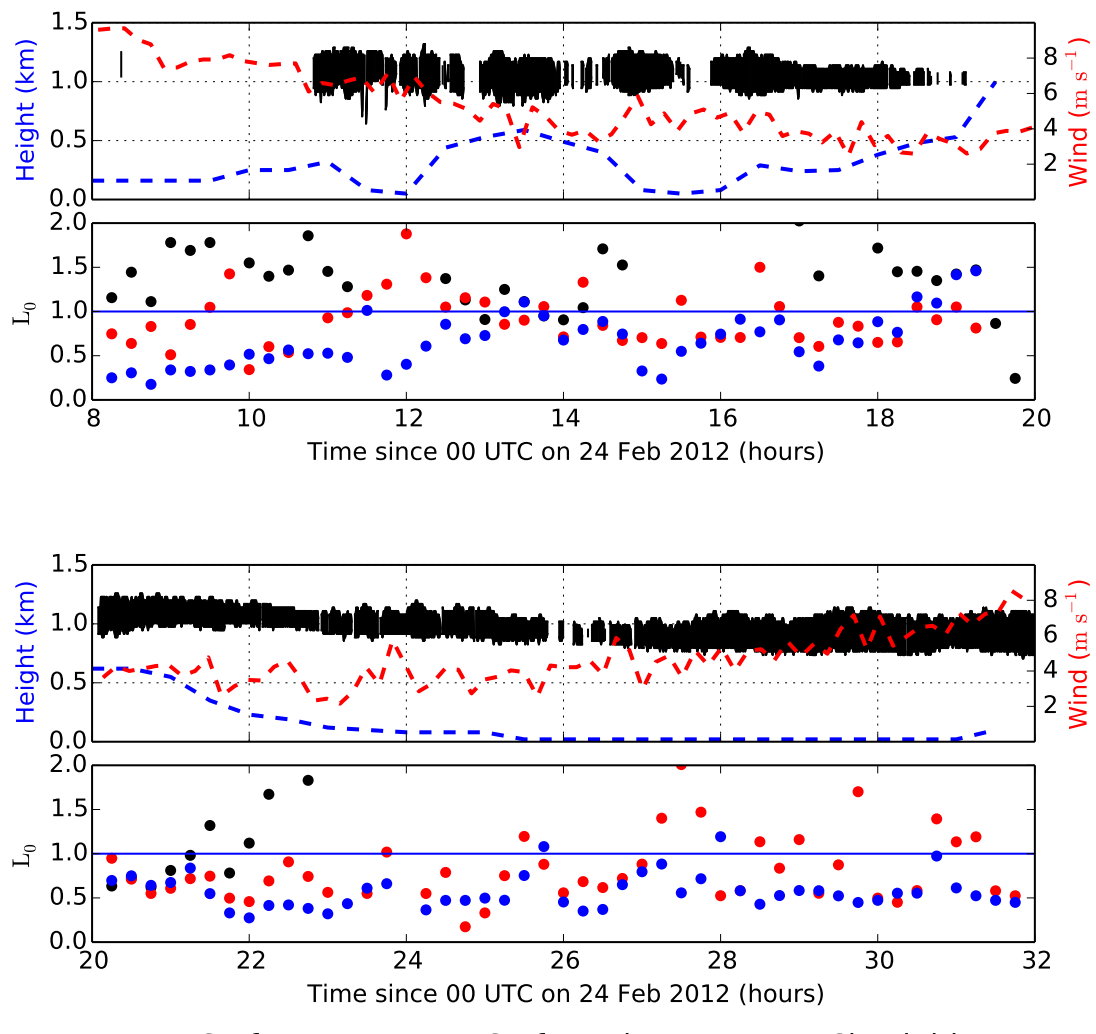

•. Surface $\quad \ldots$ Surface alt. $\quad \ldots$ Cloud driven

Figure 8. Scaling of the inertial subrange with the location of the cloud layer shown in the upper panel as the black shaded area. For this, cloud base is diagnosed from the lidar measurements, while radar measurements are used to infer cloud-top height. Blue dashed line represents an estimate of the interface between surface-based and cloud-driven mixed layers, approximated as the level at which vertical velocity skewness changes sign from positive to negative. Red dashed line shows the mean wind speed in the below-cloud layer. The lower panel shows the normalised transition wavelength of the inertial subrange $\left(L_{0}\right)$, which is sampled from the (1) cloud-driven and (2) surface-based layers, yet no lower than a $100 \mathrm{~m}$ to avoid spurious data. Two versions of the surface-layer samples are shown: "Surface" shows $L_{0}$ normalised by the surface-layer depth, while "Surface alt." shows the same data but normalised with the cloud-driven layer depth.

dar signal strength, or $L_{0}$ may be below the wavelengths resolved by the spectral decomposition. Moreover, the spectrum may sometimes feature a double-peaked structure, in which case the higher wave number peak is considered. The presentation of the results is divided into two equal-length periods ranging from 08:00 to 20:00 UTC and from 20:00 to 32:00 UTC, counting from 00:00 UTC on 24 February. In an idealised well-mixed boundary layer with isotropic turbulence one might expect to see $L_{0} \approx 1.0$, which is thus highlighted with a solid blue line in Fig. 8. This expectation arises from the first-order hypothesis that the maximum length scale of the turbulent eddies is of the same order of magnitude as the thickness of the mixed layer (Stull, 1988). As shown below, $L_{0}<1.0$ tends to indicate a suppression of the development of the turbulent mixed-layer structure. In contrast, $L_{0}>1.0$ can be interpreted as the impact of largerscale forcings, e.g. gravity-wave activity, or perhaps stretching of the turbulent eddy structure during changes in wind conditions. As seen in Fig. 8, overall $L_{0}$ varies significantly with time and with height. Yet, a distinct behaviour is ob- served with respect to the boundary layer structure and the properties of the overlying cloud deck.

During 08:00-11:00 UTC, just after the passage of the front and before the stratocumulus emerges over the site, there are clear differences in $L_{0}$ with respect to height. As the boundary layer is not yet covered by cloud during this time, the samples are drawn from roughly the heights of 100 and $600 \mathrm{~m}$. The transition wavelength $\lambda_{0}$ is considerably longer for the high-level samples than for those near the surface. This supports the analysis performed in Sect. 3, as it suggests a shallow surface boundary layer with a weakly turbulent free-tropospheric air mass above, still under synoptic influence of frontal dynamics.

As the stratocumulus layer advects over the area, $L_{0}$ is close to 1.0 in the cloud-driven regime, while in the surface layer $L_{0}$ does not present robust scaling. Normalising the data from the surface layer with the depth of the clouddriven layer reveals that the transition wavelength near the surface is, nevertheless, generally smaller than at higher levels. At noon, $L_{0}$ in the cloud-driven layer peaks to values 
close to 2.0, associated with a maximum in the depth of the layer. This coincides with the appearance of a more broken cloud structure and the growth of the surface-based layer. Between 12:00 and 15:00 UTC, $L_{0}$ from both sampling levels scales mostly very well with the corresponding mixedlayer depths, as diagnosed from the skewness. This provides a good example of the effects of decoupling on both the vertical velocity statistics and the scaling of the turbulent motions. The consistency between the scaling of the inertial subrange and decoupling height derived from skewness is well in line with the interpretation of a decoupled boundary layer outlined in Sect. 4.1. Afterwards, the skewness-based decoupling height again descends to a very low level: similar to the results from around noon, since the minimum sampling level for the surface-based layer is $100 \mathrm{~m}$, some of the lowlevel samples for $L_{0}$ may represent the cloud-driven regime. Normalising $\lambda_{0}$ from both sampling levels with the depth of the cloud-driven layer yields very similar $L_{0}$, with slightly suppressed values, i.e. mostly below 1.0. The results suggest that, during periods of pronounced separation between the surface-based and cloud-driven layers, the skewness-based decoupling height indeed appears to be a useful estimate as it agrees well with the scaling of $L_{0}$. In contrast, with lesspronounced separation in the profiles of skewness, the actual decoupling height is likely to be found at higher levels than expected, as it would explain the suppression in the clouddriven $L_{0}$; forcing $L_{0}=1.0$ in the cloud-driven layer after 15:00 UTC and inverting Eq. (5) for $\lambda_{0}$ yields a decoupling height just below $500 \mathrm{~m}$ on average, assuming that cloud top marks the boundary layer top height. This would also produce much better results for the surface-based $L_{0}$, which have values larger than 2.0 in the case of very low height of the skewness interface. In close agreement, Fig. 6 shows a marked separation in the vertical profile of dissipation rate at the height of $600 \mathrm{~m}$ after 15:00 UTC. This is actually stronger than what is seen between 12:00 and 15:00 UTC, although the opposite is true for skewness.

The situation from 20:00 UTC onwards exhibits somewhat different behaviour. From 20:00 to 32:00 UTC the clouddeck is almost continuous and there is no solar influence. The cloud-driven mixed layer grows downwards to reach the surface by 22:00 UTC and remains in the proximity of the surface until around 32:00 UTC (8 a.m. LT). During the first couple of hours (about 20:00-22:00 UTC) of the initial growth of the new cloud-driven layer, $L_{0}$ scales relatively well with the corresponding layer depths though being slightly smaller than 1.0. Towards and after midnight, $L_{0}$ from the higher sampling level gradually approaches 1.0, although it is not until 28:00 UTC that $L_{0} \approx 1.0$ (albeit the results show very large temporal variations). Since the minimum sampling height for the surface-based layer was set at $100 \mathrm{~m}$, it is expected that the normalisation by the surfacelayer depth is not going to work after midnight, since the cloud-driven mixing practically covers the entire boundary layer depth, as shown in Fig. 7. Normalising the transition wavelength from this height with the cloud-driven depth results in $L_{0}$ mostly around 0.5 , where it stays for the duration of the analysis.

Compared to the daytime period, between 18:00 and 24:00 UTC the mixing close to the surface is extremely weak. Thus, the contribution of the surface layer to the transport of TKE and the mixed-layer structure is low. The redeveloping cloud-driven mixed layer can encroach downwards quite freely during 20:00-26:00 UTC, which is clearly seen as the extending region of higher dissipation rate and vertical velocity in Fig. 4 and the negative skewness in Fig. 5. While the suppression of the cloud-driven $L_{0}$ at this time may be due to underestimated decoupling height, it may also be influenced by the low horizontal wind speeds, about $4 \mathrm{~m} \mathrm{~s}^{-1}$, which affects the wind shear and entrainment processes and thus the dimensions of the cloud-driven turbulence. Before 22:00 UTC, the surface layer shows very similar scaling of $L_{0}$ as the cloud-driven layer, despite the very weak turbulent mixing. After midnight, the samples near the surface start to represent the cloud-driven layer as well due to the minimum sampling height of $100 \mathrm{~m}$ and the increasing depth of the cloud-driven layer. Thus $\lambda_{0}$ from both sampling levels is quite similar. The gradual increase in the cloud-driven $L_{0}$ during 24:00-32:00 UTC coincides with the increase in horizontal wind speed. The intensifying wind may change the aspect ratio of the turbulent eddies by stretching the updrafts and downdrafts further apart horizontally. Additionally, changes in wind shear near the cloud top potentially modulate the entrainment process, with intense entrainment causing strong evaporative cooling. This may modify the production of turbulent energy at the top of the boundary layer (Lock, 1998), which can also affect the scaling of $L_{0}$ in the cloud-driven environment. In comparison, it should be noted that $\lambda_{0}$ near the surface does not exhibit a marked increase and corresponds to about half of the boundary layer depth.

Local sunrise is about 7:40 a.m. (31:40 UTC in Fig. 8). A new surface-driven mixed layer starts to grow, evident through positive skewness and a change in dissipation rate in Fig. 5. Although the dissipation rate (Fig. 4c) suggests that this surface-driven layer is less turbulent than the clouddriven mixed layer, it continues to grow into and erode the cloud-driven layer. Note that $L_{0}$ at all levels is abruptly reduced to 0.5 . Towards noon of 25 February the situation is under increasing influence of a gradual air mass change, explaining the reduction in cloud base height. Related to this, Fig. 2 shows evidence of an enhanced drizzle production that strongly affects the mixed-layer dynamics, making this situation very different from the earlier analysed periods.

The consequences of the variation in $L_{0}$ can be outlined by examining the advective timescales corresponding to the transition wavelength of the inertial subrange, $\lambda_{0}$. The timescales are shown in Fig. 9 and are obtained by dividing $\lambda_{0}$ by the collocated wind speed (averaged over $1 \mathrm{~h}$ and $100 \mathrm{~m}$ in the vertical). The majority of the timescales reside between 100 and $250 \mathrm{~s}$. As may be expected based on the re- 


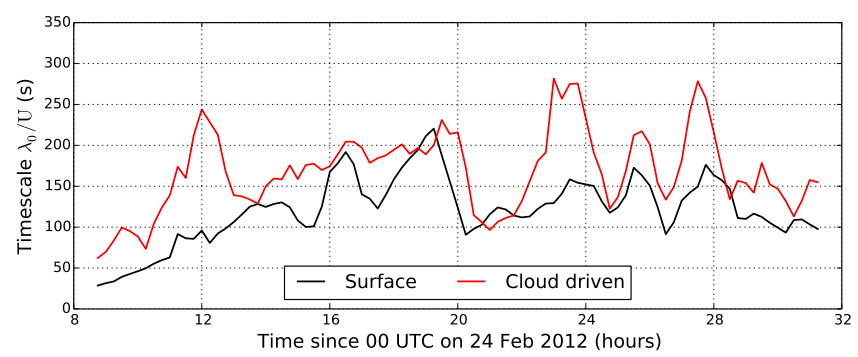

Figure 9. Advective timescales corresponding to the largest scales within the inertial subrange $\left(\lambda_{0}\right)$ shown in Fig. 8 from the surfacebased (black) and cloud-driven layers (red). $U$ denotes the horizontal wind speed.

sults above, the timescales sampled from the surface-based layer are generally somewhat smaller than those from the cloud-driven layer, especially during the peak values found for the latter. These are the result of variations not only in the cloud-driven $L_{0}$ but also in the horizontal wind. In contrast, during periods with clearly decoupled mixed-layer structure, such as that during 12:00-15:00 UTC on 24 February, the advective timescales are quite similar for the surface and clouddriven layers. Knowledge of this timescale is important when calculating derived products, such as the dissipation rate, from the lidar measurements, where it is assumed that all sampled length scales are within the inertial subrange. More samples would be preferred for more robust statistics, but as indicated in Fig. 9, complex boundary layer structures exhibit a wide variation in the length scales which reside within the inertial subrange. As an example, when deriving dissipation rate using the method of O'Connor et al. (2010), extending the sampling time beyond 3 min would imply that the spatial length scale $L$ in Eq. (6) is outside the inertial subrange, rendering the assumption used in the derivation of the equation invalid.

\section{Conclusions}

This study analysed 2 days (24-25 February 2012) of continuous high-resolution Doppler lidar observations from Mace Head, comprising a long-lived stratocumulus cloud deck following behind an overpass of a cold front.

We focused on the turbulent properties exhibited by the cloud-topped boundary layer through examining various parameters derived from the Doppler lidar vertical velocities. Power spectrum analysis of the vertical velocity was also performed to infer the range of scales of mixing associated with the inertial subrange by defining a transition wavelength normalised by the local mixed-layer depth $\left(L_{0}\right)$.

From previous studies (Hogan et al., 2009) it is known that negative skewness of vertical velocity below cloud layer indicates cloud-driven turbulent mixing e.g. due to cloud-top radiative cooling, which was present throughout the anal- ysed period. During 24 February, a broken cloud structure was observed in the stratocumulus deck, causing weaker production of turbulent kinetic energy at the top of the boundary layer. Together with decreasing horizontal winds in the afternoon of 24 February and possible impacts on the stability of the boundary layer, this decreased the depth of the cloud-driven mixed layer and allowed a weak surface-based mixed layer to grow (indicated by positive vertical velocity skewness). In effect, the cloud deck was decoupled from the surface, although the degree of decoupling was likely moderate, based on the profiles of turbulence statistics. This was supported also by the LCL estimated from surface measurements of temperature and humidity. In contrast, during the night, the degree of decoupling was much lower. The mixing was still cloud-driven and the mixed layer grew to encompass almost the entire depth of the boundary layer. In this case, because the surface-generated TKE was generally low, the extent of the cloud-driven layer depends essentially on the stability conditions and the intensity of TKE generation in the cloud layer, which was strongest during the night.

The investigation of the normalised transition wavelength $L_{0}$ through spectral analysis showed that $L_{0}$ scales relatively well with the corresponding mixed-layer depth diagnosed from skewness, especially in the cloud-driven layer. The decoupling height between the cloud-driven and the surface layer was estimated as the level where the vertical velocity skewness changes sign from positive to negative, as ascending towards the cloud deck. When a marked separation between the mixed layers was present with a relatively high decoupling height as diagnosed from the profiles of skewness, $L_{0}$ in both the surface-based and cloud-driven layers scaled very well with the corresponding layer depths. This agreement between the vertical velocity statistics and the scaling of the inertial subrange corroborates the use of vertical velocity skewness as an indicator of the boundary layer structure. However, in many occasions when the surface-based region of positive vertical velocity skewness was less pronounced and the estimated decoupling height was consequently reduced, scaling of $L_{0}$ generally suggested a considerably higher decoupling height than expected based on the profile of skewness.

In comparison, periods with a well-developed coupled (yet cloud-driven) nocturnal mixed layer showed $L_{0} \approx 1.0$ in the cloud-driven layer, following the intensification of the horizontal wind. Before this, $L_{0}$ stayed relatively low. This shift is likely the result of shear stress affecting the geometry of the turbulent eddies with increasing wind and also the production of turbulent kinetic energy at cloud top due to changes in entrainment. However, near the surface $L_{0}$ remained suppressed throughout this period.

The results from this campaign show that power spectral analysis of vertical velocity from continuously operated lidars can be used to identify and verify the existence of decoupled mixed layers within the boundary layer. These results are also partially in agreement with earlier studies using 
profiles of vertical velocity skewness to identify the different mixed-layer regimes, although for weakly turbulent surface layers the vertical velocity statistics were not always robust indicators for the actual decoupling height. Nevertheless, vertically resolved $L_{0}$ from continuously operating Doppler lidars provides an additional tool to diagnose the structural features of complex cloud-topped boundary layers and complements the use of statistics profiles in diagnosing the decoupling height. In addition, the identification of potential rapid variations in $\lambda_{0}$ and the reductions seen in decoupled situations are an important consideration when calculating products such as turbulent dissipation rate because of the resulting constraints on the sampling interval for deriving these parameters.

Acknowledgements. This work has been supported by a Väisälä foundation grant from the Finnish Academy of Science and Letters as well as the ERC project PBL-PMES (grant number 227915). Financial support from the Academy of Finland (project 283030) and the Centre of Excellence Programme (project 1118615 ) is acknowledged. The research leading to these results has also received funding from the European Union Seventh Framework Programme (FP7/2007-2013) under grant agreement no. 262254. We thank ACTRIS Transnational Access, the Irish EPA and the Irish Higher Education Authority for their support. Three reviewers are acknowledged for their constructive comments.

Edited by: G. Feingold

\section{References}

Babb, D. M. and Verlinde, J.: Vertical velocity statistics in continental stratocumulus as measured by a $95 \mathrm{GHz}$ radar, Geophys. Res. Lett., 86, 1177-1180, 1999.

Banakh, V. A., Smalikho, I. N., Köpp, F., and Werner, C.: Measurements of Turbulent Energy Dissipation Rate with a CW Doppler Lidar in the Atmospheric Boundary Layer, J. Atmos. Ocean. Tech., 16, 1044-1061, 1999.

Christensen, M. W., Carrió, G. G., Stephens, G. L., and Cotton, W. R.: Radiative impacts of free-tropospheric clouds on the properties of marine stratocumulus, J. Atmos. Sci., 70, 31023118, doi:10.1175/JAS-D-12-0287.1, 2013.

Duynkerke, P. G., Zhang, H., and Jonker, P. J.: Microphysical and turbulent structure of nocturnal stratocumulus as observed during ASTEX, J. Atmos. Sci., 52, 2763-2777, 1995.

Frisch, A. S., Lenschow, D. H., Fairall, C. W., Schubert, W. H., and Gibson, J. S.: Doppler radar measurements of turbulence in marine stratiform cloud during ASTEX, J. Atmos. Sci., 52, 28002808, 1995.

Gal-Chen, T., Xu, M., and Eberhard, W. L.: Estimations of atmospheric boundary layer fluxes and other turbulence parameters from Doppler lidar data, J. Geophys. Res., 97, 18409-18423, 1992.

Garrat, J. R.: The Atmospheric Boundary Layer, Cambridge University Press, New York, USA, 316 pp., 1992.
Ghate, V. P., Albrecht, B. A., Miller, M. A., Brewer, A., and Fairall, C. W.: Turbulence and radiation in stratocumulus-topped marine boundary layers: A case study from VOCALS-REx J. Appl. Meteor. Climatol., 53, 117-135, doi:10.1175/JAMC-D-12-0225.1, 2014.

Gossard, E. E., Wolfe, D. E., Moran, K. P., Paulus, R. A., Anderson, K. D., and Rogers, L. T.: Measurement of clear-air gradients and turbulence properties with radar wind profilers, J. Atmos. Ocean. Tech., 15, 321-342, 1998.

Harvey, N. J., Hogan, R. J., and Dacre, H. F.: A method to diagnose boundary-layer type using Doppler lidar, Q. J. Roy. Meteor. Soc., 139, 1681-1693, doi:10.1002/qj.2068, 2013.

Hirsikko, A., O’Connor, E. J., Komppula, M., Korhonen, K., Pfüller, A., Giannakaki, E., Wood, C. R., Bauer-Pfundstein, M., Poikonen, A., Karppinen, T., Lonka, H., Kurri, M., Heinonen, J., Moisseev, D., Asmi, E., Aaltonen, V., Nordbo, A., Rodriguez, E., Lihavainen, H., Laaksonen, A., Lehtinen, K. E. J., Laurila, T., Petäjä, T., Kulmala, M., and Viisanen, Y.: Observing wind, aerosol particles, cloud and precipitation: Finland's new groundbased remote-sensing network, Atmos. Meas. Tech., 7, 13511375, doi:10.5194/amt-7-1351-2014, 2014.

Hogan, R. J., Grant, A. L., Illingworth, A. J., Pearson, G. N., and O'Connor, E. J.: Vertical velocity variance and skewness in clear and cloud-topped boundary layers as revealed by Doppler lidar, Q. J. Roy. Meteor. Soc., 135, 635-643, doi:10.1002/qj.413, 2009.

Jacoby-Koaly, S., Campistron, B., Bernard, S., Benech, B., Ardhuin-Girard, F., Dessens, J., Dupont, E., and Carissimo, B.: Turbulent dissipation rate in the boundary layer via UHF wind profiler Doppler spectral width measurements, Bound.-Lay. Meteorol., 103, 361-389, 2002.

Kollias, P. and Albrecht, B.: The turbulence structure in continental stratocumulus cloud from millimeter-wavelength radar observations, J. Atmos. Sci., 57, 2417-2433, 2000.

Koscielny, A. D., Doviak, R. J., and Zrnić , D. S.: An evaluation of the accuracy of some radar wind profiling techniques, J. Atmos. Ocean. Tech., 1, 309-320, 1984.

Kouznetsov, R., Kramar, V. F., and Kallistratova, M. A.: The vertical structure of turbulent momentum flux in the lower part of the atmospheric boundary layer, Meteorol. Z., 16, 367-373, 2007.

Kristensen, L., Lenschow, D. H., Kirkegaard, P., and Courtney, M.: The spectral velocity tensor for homogeneous boundary layer turbulence, Bound.-Lay. Meteorol., 47, 149-193, 1989.

Lawrence, M. G.: The relationship between relative humidity and the dewpoint temperature in moist air - a simple conversion and applications. Bull. Am. Meteorol. Soc, 86, 225-233, doi:10.1175/BAMS-86-2-225, 2005.

Lock, A. P.: The parameterization of entrainment in cloudy boundary layers, Q. J. Roy. Meteor. Soc., 124, 2729-2753, 1998.

Lock, A. P., Brown, A. R., Bush, M. R., Martin, G. M., and Smith, R. N. B.: A new boundary layer mixing scheme. Part I: Scheme description and single-column model tests, Mon. Weather Rev., 128, 3187-3199, 2000.

Lothon, M., Lenschow, D. H., and Mayor, S. D.: Coherence and scale of vertical velocity in the convective boundary layer from a Doppler lidar, Bound.-Lay. Meteorol., 121, 521-536, doi:10.1007/s10546-006-9077-1, 2006.

Lothon, M., Lenschow, D. H., and Mayor, S. D.: Doppler lidar measurements of vertical velocity spectra in the convective plan- 
etary boundary layer, Bound.-Lay. Meteorol., 132, 205-226, doi:10.1007/s10546-009-9398-y, 2009.

Moyer, K. A. and Young, G. S.: Observations of vertical velocity skewness within the marine stratocumulus-topped boundary layer, J. Atmos. Sci., 48, 403-410, 1991.

Nicholls, S.: The dynamics of stratocumulus: aircraft observations and comparisons with a mixed layer model, Q. J. Roy. Meteor. Soc., 110, 783-820, 1984.

Nicholls, S.: The structure of radiatively driven convection in stratocumulus, Q. J. Roy. Meteor. Soc., 115, 487-511, 1989.

Norton, E. G., Vaughan, G., Methven, J., Coe, H., Brooks, B., Gallagher, M., and Longley, I.: Boundary layer structure and decoupling from synoptic scale flow during NAMBLEX, Atmos. Chem. Phys., 6, 433-445, doi:10.5194/acp-6-433-2006, 2006.

O'Connor, E. J., Illingworth, A. J., and Hogan, R. J.: A technique for autocalibration of cloud lidar, J. Atmos. Ocean. Tech., 21, 777-786, 2004.

O’Connor, E. J., Illingworth, A. J., Brooks, I. M., Westbrook, C. D., Hogan, R. J., Davies, F., and Brooks, B. J.: A method for estimating the turbulent kinetic energy dissipation rate from a vertically pointing Doppler lidar, and independent evaluation from balloon-borne in situ measurements, J. Atmos. Ocean. Tech., 27, 1652-1664, doi:10.1175/2010JTECHA1455.1, 2010.
Rye, B. J. and Hardesty, R. M.: Discrete spectral peak estimation in incoherent backscatter heterodyne lidar. I: Spectral accumulation and the Cramer-Rao lower bound, IEEE T. Geosci. Remote, 31, 16-27, 1993.

Shupe, M. D., Brooks, I. M., and Canut, G.: Evaluation of turbulent dissipation rate retrievals from Doppler Cloud Radar, Atmos. Meas. Tech., 5, 1375-1385, doi:10.5194/amt-5-1375-2012, 2012.

Stull, R. B.: An Introduction to Boundary Layer Meteorology, Kluwer Academic Publishers, Dordrecht, the Netherlands, 666 pp., 1988.

Westbrook, C. D., Illingworth, A. J., O'Connor, E. J., and Hogan, R. J.: Doppler lidar measurements of oriented planar ice crystals falling from supercooled and glaciated cloud layers, Q. J. Roy. Meteor. Soc., 136, 260-276, 2010. 